PROCEEDINGS OF THE

AMERICAN MATHEMATICAL SOCIETY

Volume 135, Number 6, June 2007, Pages 1935-1945

S 0002-9939(07)08734-5

Article electronically published on February 9, 2007

\title{
ON EXCEEDANCES OF RECORD AND ORDER STATISTICS
}

\author{
I. G. BAIRAMOV AND M. K. KHAN \\ (Communicated by Edward C. Waymire)
}

\begin{abstract}
Exact, limiting distributions along with their rates of convergence of exceedance statistics for both order statistics and record statistics are provided when the underlying distribution is arbitrary. The exact distribution of record statistics for arbitrary underlying distributions is obtained as well.
\end{abstract}

\section{INTRODUCTION}

Let $X_{1}, X_{2}, \ldots, X_{n}, \ldots$ be a sequence of iid random variables with distribution function $F$ which belongs to some class of distributions $\mathbb{F}$. Let $a_{n}:=a\left(x_{1}, x_{2}, \ldots, x_{n}\right)$ and $b_{n}:=b\left(x_{1}, x_{2}, \ldots, x_{n}\right)$ be two Borel functions in $\Re^{n}$ satisfying $a_{n} \leq b_{n}$ over $\Re^{n}$. Let $\nu_{m}$ be the number of $X_{n+1}, X_{n+2}, \ldots, X_{n+m}$ falling in the interval $\left(a_{n}, b_{n}\right)$ which is constructed from the observations of $X_{1}, X_{2}, \ldots, X_{n}$. Evidently $\nu_{m}$ is the sum of dependent binary random variables and its distribution depends on the probability

$$
P\left\{X_{n+1} \in\left(a_{n}, b_{n}\right)\right\} .
$$

Some authors call this model a generalized Bernoulli model. If $\mathbb{F}=\mathbb{F}_{c}$ is the class of all continuous distribution functions, then the probability (1.1) is the same for all $F \in \mathbb{F}_{c}$ if and only if $a_{n}=a\left(X_{1}, X_{2}, \ldots, X_{n}\right)=X_{i: n}$ and $b_{n}=$ $b\left(X_{1}, X_{2}, \ldots, X_{n}\right)=X_{j: n}$ for some $i$ and $j(1 \leq i<j \leq n)$, where $X_{1: n} \leq$ $X_{2: n} \leq \ldots \leq X_{n: n}$ are the order statistics constructed from the first observations $X_{1}, X_{2}, \ldots, X_{n}$. Furthermore, in this case (see Bairamov and Petunin [7]), $P\left\{X_{n+1} \in\left(X_{i: n}, X_{j: n}\right)\right\}=\frac{j-i}{n+1}$. A random interval having such a property is called an invariant confidence interval containing the future observations. If $\left(a_{n}, b_{n}\right)$ is an invariant interval, then $\nu_{m}$ is a distribution free statistic for the class $\mathbb{F}$. A similar property is valid for record values. Let $X_{U(n)}$ denote the $r$-th record value and let $X_{U(n)+1}, X_{U(n)+2}, \ldots, X_{U(n)+m}$ be the next $m$ observations that come after $X_{U(n)}$. It is easy to show that $X_{U(n)}, X_{U(n)+1}, X_{U(n)+2}, \ldots, X_{U(n)+m}$ are mutually independent random variables and $X_{U(n)+i}, i=1,2, \ldots, m$, has the same distribution function $F$. When $F$ is absolutely continuous, Bairamov 3 proved that $P\left\{X_{U(s)+1} \in\left(X_{U(r)}, X_{U(s)}\right)\right\}=\frac{1}{2^{r}}-\frac{1}{2^{s}}, r<s$. A statistic connected with the number of observations exceeding the random threshold is called an exceedance statistic. Several authors have considered $X_{r: n}(1 \leq r \leq n)$ and $X_{U(n)}, n>1$, as random thresholds and studied the distributional properties of the corresponding exceedance

Received by the editors July 27, 2004 and, in revised form, March 27, 2006.

2000 Mathematics Subject Classification. Primary 62G30; Secondary 62E20.

Key words and phrases. Exact distributions, limit theorems, rates of convergence, BerryEsseen bounds. 
statistics and their applications. More precisely, let $X_{1}, X_{2}, \ldots, X_{n}$ be a sample with distribution function $F_{X}$ and let $Y_{1}, Y_{2}, \ldots, Y_{m}$ be another sample, independent of the first one, having distribution function $F_{Y}$. It is clear that if $H_{0}: F_{X}=F_{Y}$ is true, then $Y_{1}, Y_{2}, \ldots, Y_{m}$ can be considered as $X_{n+1}, X_{n+2}, \ldots, X_{n+m}$, i.e., as a continuation of $X_{1}, X_{2}, \ldots, X_{n}$. In the case of continuous underlying distribution functions, Katzenbeisser [15, [16 obtained an expression for the distribution of $\nu_{m}$ when $\left(a\left(X_{1}, X_{2}, \ldots, X_{n}\right), b\left(X_{1}, X_{2}, \ldots, X_{n}\right)\right)=\left(X_{i: n}, X_{j: n}\right), 1 \leq i<j \leq n$, and proposed test criteria for testing the null hypothesis $H_{0}: F_{X}=F_{Y}$ versus the Lehman alternatives, $F_{Y}(x)=\left[F_{X}(x)\right]^{\theta}, \theta \neq 1$. Matveychuk and Petunin [17, 18, and Johnson and Kotz [13, 14, studied a generalized Bernoulli model in terms of placement statistics from two random samples and suggested test criteria for testing the hypothesis $H_{0}: F_{X}=F_{Y}$ using $\nu_{m}$. On the other hand, Bairamov 3 considered record threshold models and derived exact and asymptotic distributions of some exceedance statistics. For more on these developments see [20, 4, 8], 1], [2].

The results mentioned above are for the case when the underlying distributions are absolutely continuous. The main point of departure of this paper is that we keep the underlying distribution arbitrary. Until now even the distribution of the $r$ th-order record value has not been characterized when $F$ is arbitrary. Recently Bairamov and Kotz [6] considered the case when the underlying distribution had isolated atoms, $a_{1}, a_{2}, \ldots, a_{n}$, where $F\left(a_{i}-0\right)<F\left(a_{i}\right), i=1,2, \cdots, n$, and $F$ is continuous otherwise. They studied the distributional properties of exceedance statistics when the threshold is the $r$ th-order statistic or the second record value.

In this paper we study distributions of exceedances associated with order statistics and record values for arbitrary distributions. We derive the exact distribution of the $r$ th-record value for arbitrary underlying distributions, which was not available to date. The limit distributions, along with their rates, for exceedances of record and order statistics are characterized. The main results are collected in the next section and their proofs are provided in section 3 .

\section{The MAIN RESUlts}

Let $X_{1}, X_{2}, \cdots$ be a sequence of independent and identically distributed random variables with distribution $F$ of arbitrary type and let $D$ be the set of points of discontinuity of $F$. Throughout $X_{1: n} \leq X_{2: n} \leq \cdots \leq X_{n: n}$ will represent the order statistics from a sample of size $n$ and $F_{r: n}$ will be the distribution of $X_{r: n}$. For a second sample, $X_{n+1}, \cdots, X_{n+m}$, define the exceedance statistic, $S_{m}(r)$, to be the number of $X_{n+i}$ that fall strictly below $X_{r: n}$. (We should remark that one may modify our results if exceedance is defined as $X_{n+i} \leq X_{r: n}$; however, we will not duplicate such results.) The sampling distribution of $S_{m}(r)$ is as follows.

Theorem 2.1. For $k=0,1, \cdots, m$, we have

$$
\begin{array}{r}
\frac{P\left(S_{m}(r)=k\right)}{\left(\begin{array}{c}
m \\
k
\end{array}\right)}=\frac{r\left(\begin{array}{l}
n \\
r
\end{array}\right)}{(m+n)\left(\begin{array}{c}
m+n-1 \\
k+r-1
\end{array}\right)}\left(1-\sum_{d \in D}\left(\Delta F_{k+r: m+n}\right)_{d}\right) \\
+\sum_{d \in D}\left(F\left(d^{-}\right)\right)^{k}\left(1-F\left(d^{-}\right)\right)^{m-k}\left(\Delta F_{r: n}\right)_{d},
\end{array}
$$

where $\left(\Delta F_{r: n}\right)_{d}=F_{r: n}(d)-F_{r: n}\left(d^{-}\right)$, and $\sum_{d \in D}$ stands for summing over all points of discontinuity of $F$. 
When $D$ is empty, one gets the classical result of Gumbel and von Schelling [12], see also Gumbel [10, 11], Epstein [9, and Sarkadi [19]. When $D$ is a finite set, one gets the recent result of Bairamov and Kotz [6].

Our next result deals with the weak convergence of the statistic $S_{m}(r) / m$. When $F$ is a continuous distribution, the weak convergence of $S_{m}(r) / m$ to a beta distribution was proved by Matverchuk and Petunin [17, [18. For the record exceedance model, the weak convergence, when the underlying distribution is continuous, was proved by Bairamov [3]. Bairamov et. al. [5] derived the limiting distribution of $S_{m}(r)$ falling in $\left(a_{n}, b_{n}\right)$ when $a_{n}, b_{n}$ are symmetric functions of their arguments. The following theorem not only provides the weak limit of $S_{m}(r) / m$ for an arbitrary $F$ but also gives its rate of convergence.

Theorem 2.2. For any distribution $F$, and any $x \in(0,1)$, the rate of weak convergence is

$$
\left|P\left(S_{m}(r) \leq m x\right)-\widehat{L_{r, n}}(x)\right|=O\left(m^{-1 / 2}\right)
$$

where

$$
\widehat{L_{r, n}}(x):= \begin{cases}B_{r, n-r+1}(F(d)) & \text { if } x \in\left(F\left(d^{-}\right), F(d)\right] \text { for some } d \in D, \\ \frac{B_{r, n-r+1}(F(d))+B_{r, n-r+1}\left(F\left(d^{-}\right)\right)}{2} & \text { if } x=F\left(d^{-}\right) \text {for some } d \in D, \\ B_{r, n-r+1}(x) & \text { otherwise, }\end{cases}
$$

and where $B_{a, b}(x)$ is the beta distribution with parameters a,b. (Of course, the weak limit is the right continuous version of $\widehat{L_{r, n}}$.)

In particular, when $F$ is a continuous distribution, we get

$$
\left|P\left(S_{m}(r) \leq m x\right)-B_{r, n-r+1}(x)\right|=O\left(m^{-1 / 2}\right) .
$$

Since $B_{r, n-r+1}(x)$ is a continuous distribution, by Polya's theorem, weak convergence of $S_{m}(r) / m$ to $B_{r, n-r+1}(x)$ takes place uniformly in $x$.

Next, we identify the exact distribution of record values when the underlying distribution is arbitrary. Let $U(1)=1$ and let $U(n)=\min \left\{i: i>U(n-1), X_{i}>\right.$ $\left.X_{U(i-1)}\right\}$ for $n>1$ be the record times and let $X_{U(1)}, X_{U(2)}, \cdots$ be the corresponding record values. In our notation $X_{U(1)}=X_{1}$ is the trivial record value and $X_{U(2)}$ is the first nontrivial record value. Note that if $F$ is such that there is a point $d$ so that $F(d)=1$ and $F\left(d^{-}\right)<1$, then with positive probability, a record could get "stuck" forever. Due to this reason, throughout we will assume that if $F(x)=1$ for some real number $x$, then $x$ is a point of continuity.

When $X_{1}$ has an absolutely continuous distribution function, $F$, it is easy to see that the distribution of the first nontrivial record value is $F(x)+(1-F(x)) \ln (1-$ $F(x)$ ). The following theorem gives the distribution of the first nontrivial record value for an arbitrary distribution $F$.

Theorem 2.3. The distribution $H_{2}(x):=P\left(X_{U(2)} \leq x\right)$ is as follows:

$$
H(F(x))-\sum_{d \leq x}\left\{H(F(d))-H\left(F\left(d^{-}\right)\right)-R\left(F\left(d^{-}\right)\right)(\Delta F)_{d}\right\} \frac{1-F(x)}{1-F(d)},
$$

where $H(w)=w+(1-w) \ln (1-w), R(w)=-\ln (1-w),(\Delta F)_{d}=F(d)-F\left(d^{-}\right)$ and $\sum_{d \in A}$ stands for summing over all points of discontinuity of $F$ in $A$. 
For the distribution of higher-order record values, we need some notation. Let

$R_{2}(x):=\int_{(-\infty, x]} \frac{1}{1-F(u)} d F(u), \quad R_{r}(x):=\int_{(-\infty, x]} \frac{R_{r-1}\left(u^{-}\right)}{1-F(u)} d F(u), \quad x \in \Re$,

for $r=3,4, \cdots$. Let $f_{r}(x)=(-\ln (1-x))^{r-1} /(r-1)$ ! for $x \in[0,1)$ and zero otherwise, and let $\phi_{r}$ be its cumulative distribution function. For $d<x$, let $h_{2}(x, d) \equiv 1$, $h_{2}(x, x)=0$, and

$$
\begin{aligned}
h_{r}(x, d) & :=\int_{(d, x]} \frac{h_{r-1}\left(u^{-}, d\right)}{1-F(u)} d F(u), \quad r=3,4, \cdots, \quad d \leq x, \\
g_{r}(d) & :=\left\{f_{r}^{\prime} \circ F(d) \frac{f_{r-1} \circ F\left(d^{-}\right)}{f_{r-1} \circ F(d)}-\frac{\left(\Delta f_{r} \circ F\right)_{d}}{(\Delta F)_{d}}\right\}(\Delta F)_{d}, \quad r=2,3, \cdots, \quad d \in D .
\end{aligned}
$$

Theorem 2.4. The Radon-Nikodym derivative of $H_{r}(x):=P\left(X_{U(r)} \leq x\right)$, with respect to $F$, is $R_{r}\left(x^{-}\right)$. Furthermore,

$$
\begin{aligned}
& R_{r}(x)=f_{r} \circ F(x)+\sum_{d \leq x} \sum_{k=2}^{r} g_{k}(d) h_{r-k+2}(x, d), \quad r=2,3, \cdots, \\
& H_{r}(x)=\phi_{r} \circ F(x)+G_{r}(x)+\sum_{d \leq x} \sum_{k=2}^{r} g_{k}(d) \int_{(d, x]} h_{r-k+2}\left(u^{-}, d\right) d F(u),
\end{aligned}
$$

where $G_{r}(x):=\sum_{d \leq x}\left(f_{r} \circ F\left(d^{-}\right)(\Delta F)_{d}-\left(\Delta \phi_{r} \circ F\right)_{d}\right)$.

Similar expressions for the joint distribution of record statistics also hold. For any integer $r \geq 1$, let $\eta_{i}=1$ if $X_{U(r)+i}<X_{U(r)}$ and zero otherwise, and let $S_{m}^{*}(r)=\sum_{i=1}^{m} \eta_{i}$ be the exceedance from the $r$ th record. For any $x \in(0,1)$, the limiting function $S_{m}^{*}(r) / m$ is defined as follows:

$$
\widehat{M}_{r}(x):= \begin{cases}H_{r}(d) & \text { if for some } d \in D, x \in\left(F\left(d^{-}\right), F(d)\right], \\ \frac{H_{r}(d)+H_{r}\left(d^{-}\right)}{H_{r}\left(s_{x}\right)} & \text { if for some } d \in D, x=F\left(d^{-}\right) \\ & \text {otherwise, where } s_{x}=\sup \{t: F(t) \leq x\}\end{cases}
$$

The right continuous version of $\widehat{M}_{r}(x)$ is the weak limit of $S_{m}^{*}(r) / m$ with the following rate.

Theorem 2.5. For any $x \in(0,1)$, we have

$$
\left|P\left(S_{m}^{*}(r) \leq m x\right)-\widehat{M}_{r}(x)\right|=O\left(m^{-1 / 2}\right) .
$$

In particular, when $F$ is continuous, $S_{m}^{*}(r) / m$ converges weakly (uniformly in $x$ ) to $\phi_{r}(x)$. The exact distribution of $S_{m}^{*}(r)$ can also be obtained; however, the expressions get somewhat messy. An explicit expression of the distribution of $S_{m}^{*}(2)$, for instance, is provided below. 
Theorem 2.6. Using the notation of Theorem (2.3), for $\ell=0,1,2, \cdots, m$, the probability $P\left(S_{m}^{*}(2)=\ell\right)$ is given by

$$
\begin{aligned}
& \frac{P\left(S_{m}^{*}(2)=\ell\right)}{\left(\begin{array}{c}
m \\
\ell
\end{array}\right)}=\int_{0}^{1} u^{\ell}(1-u)^{m-\ell} d \phi_{2}(u)-\sum_{d \in D} \int_{F\left(d^{-}\right)}^{F(d)} u^{\ell}(1-u)^{m-\ell} d \phi_{2}(u) \\
& \quad+\sum_{d \in D}\left(F\left(d^{-}\right)\right)^{\ell}\left(1-F\left(d^{-}\right)\right)^{m-\ell}(\Delta F)_{d} R\left(F\left(d^{-}\right)\right) \\
& \quad+\sum_{d \in D}\left(-(\Delta R(F(.)))_{d}+\frac{(\Delta F)_{d}}{1-F(d)}\right)\left[\int_{F(d)}^{1} u^{\ell}(1-u)^{m-\ell} d u\right. \\
& \left.\quad-\sum_{\delta>d} \int_{F\left(\delta^{-}\right)}^{F(\delta)} u^{\ell}(1-u)^{m-\ell} d u+\sum_{\delta>d}\left(F\left(\delta^{-}\right)\right)^{\ell}\left(1-F\left(\delta^{-}\right)\right)^{m-\ell}(\Delta F)_{\delta}\right] .
\end{aligned}
$$

3. THE PROOFS

Proof of Theorem 2.1. Let $A_{n+i}=\left\{X_{n+i}<X_{r: n}\right\}$, and let $D$ be the set of points of discontinuity of $F$, and use the notation

$$
K_{D}:=\sum_{d \in D}\left(F\left(d^{-}\right)\right)^{k}\left(1-F\left(d^{-}\right)\right)^{m-k}\left(\Delta F_{r: n}\right)_{d} .
$$

Note that $F_{r: n}$ is absolutely continuous with respect to $F$ with derivative

$$
\frac{d F_{r: n}}{d F}(u)=\frac{F^{r-1}(u)(1-F(u))^{(n-r+1)-1}}{B(r, n-r+1)}=b_{r, n-r+1}(F(u)), \quad u \in D^{c},
$$

where $b_{r, n-r+1}(x)$ is the density of $B_{r, n-r+1}(x)$. Hence, we have

$$
\begin{aligned}
\frac{P\left(S_{m}(r)=k\right)}{\left(\begin{array}{c}
m \\
k
\end{array}\right)}=P\left(A_{n+1} \cap \cdots \cap A_{n+k} \cap A_{n+k+1}^{c} \cap \cdots \cap A_{n+m}^{c}\right) \\
=\int_{\Re}\left(F\left(t^{-}\right)\right)^{k}\left(1-F\left(t^{-}\right)\right)^{m-k} d F_{r: n}(t) \\
=\int_{D^{c}}\left(F\left(t^{-}\right)\right)^{k}\left(1-F\left(t^{-}\right)\right)^{m-k} d F_{r: n}(t)+K_{D} \\
=\frac{1}{B(r, n-r+1)} \int_{D^{c}}(F(t))^{k+r-1}(1-F(t))^{m-k+n-r} d F(t)+K_{D} \\
=\frac{B(k+r, m-k+n-r+1)}{B(r, n-r+1)} \int_{\Re} 1 d F_{k+r: m+n}(t) \\
\quad-\frac{B(k+r, m-k+n-r+1)}{B(r, n-r+1)} \sum_{d \in D}\left(\Delta F_{k+r: m+n}\right)_{d}+K_{D} .
\end{aligned}
$$

Simplifying the beta functions finishes the proof.

Proof of Theorem 2.2. The proof depends on three cases: namely, (i) when $x \in$ $\left(F\left(d^{-}\right), F(d)\right]$ for some $d \in D$, or (ii) when $x=F\left(d^{-}\right)$for some $d \in D$, or (iii) otherwise. The arguments being similar, we only provide the details in the first case. So let there exist a $d \in D$ so that $x \in\left(F\left(d^{-}\right), F(d)\right]$, and let $A_{x}:=\{t$ : $\left.F\left(t^{-}\right)<x\right\}=(-\infty, d]$. We may write

$$
\widehat{L_{r, n}}(x)=\int_{\left\{t: F\left(t^{-}\right)<x\right\}} 1 d B_{r, n-r+1}(F(t)) .
$$


If $S_{m, p}$ is a binomial random variable with parameters $m, p$, we may write

$$
\begin{aligned}
P( & \left.S_{m}(r) \leq m x\right)-\widehat{L_{r, n}}(x)=\int_{(-\infty, d]}\left(P\left(S_{m, F\left(t^{-}\right)} \leq m x\right)-1\right) d B_{r, n-r+1}(F(t)) \\
& \quad+\int_{\left\{t: F\left(t^{-}\right)>x\right\}} P\left(S_{m, F\left(t^{-}\right)} \leq m x\right) d B_{r, n-r+1}(F(t)) \\
= & \left(\int_{C_{1}}+\int_{C_{2}}+\int_{C_{3}}\right) P\left(S_{m, F\left(t^{-}\right)}>m x\right) d B_{r, n-r+1}(F(t)) \\
=: & A^{(1)}+A^{(2)}+B,
\end{aligned}
$$

where $C_{1}=\left\{t: \frac{x}{2}<F\left(t^{-}\right)<x\right\}, C_{2}=\left\{t: F\left(t^{-}\right) \leq \frac{x}{2}\right\}, C_{3}=\left\{t: F\left(t^{-}\right)>x\right\}$. Let $\sigma^{2}(x)=x(1-x)$ for $x \in[0,1]$. For $A^{(2)}$ just use Chebyshev's inequality:

$$
A^{(2)} \leq \int_{\left\{t: F\left(t^{-}\right) \leq \frac{x}{2}\right\}} \frac{\sigma^{2}\left(F\left(t^{-}\right)\right)}{m(x / 2)^{2}} d B_{r, n-r+1}(F(t))=O(1 / m) .
$$

Throughout $Z \sim N(0,1)$ with cumulative distribution function $\Phi$. Apply the BerryEsseen bound to get

$$
\begin{aligned}
& \int_{\left\{t: \frac{x}{2}<F\left(t^{-}\right)<x\right\}}\left|P\left(S_{m, F\left(t^{-}\right)}>m x\right)-P\left(Z>\frac{\sqrt{m}\left(x-F\left(t^{-}\right)\right)}{\sigma\left(F\left(t^{-}\right)\right)}\right)\right| d B_{r, n-r+1}(F(t)) \\
& \quad \leq \frac{C}{c_{x} \sqrt{m}} \int_{\left\{t: \frac{x}{2}<F\left(t^{-}\right)<x\right\}} 1 d B_{r, n-r+1}(F(t)),
\end{aligned}
$$

where $c_{x}$ is the minimum of $\sigma\left(F\left(t^{-}\right)\right)$over $\left\{t: \frac{x}{2}<F\left(t^{-}\right)<x\right\}$. Since $x \in$ $\left(F\left(d^{-}\right), F(d)\right]$, we have $x-F\left(d^{-}\right)>0$. Hence, the Chebyshev inequality gives

$$
\begin{aligned}
A^{(1)} & \leq O\left(m^{-1 / 2}\right)+\int_{\left\{t: F\left(t^{-}\right)<x\right\}} P\left(Z>\frac{\sqrt{m}\left(x-F\left(t^{-}\right)\right)}{\sigma\left(F\left(t^{-}\right)\right)}\right) d B_{r, n-r+1}(F(t)) \\
& \leq O\left(m^{-1 / 2}\right)+\frac{E|Z|}{\left(x-F\left(d^{-}\right)\right) \sqrt{m}} .
\end{aligned}
$$

The argument is mostly similar for $B$ and we omit some of the details, except the case when $x=F(d)$ in which case a different argument is needed. Again,

$$
B=\left(\int_{C_{4}}+\int_{C_{5}}\right) P\left(S_{m, F\left(t^{-}\right)} \leq m x\right) d B_{r, n-r+1}(F(t))=: B^{(1)}+B^{(2)},
$$

where $C_{4}=\left\{t: x<F\left(t^{-}\right)<\frac{1+x}{2}\right\}, C_{5}=\left\{t: \frac{1+x}{2} \leq F\left(t^{-}\right)\right\}$. For $B^{(2)}$ the Chebyshev inequality gives the rate. For $B^{(1)}$, the Berry-Esseen bound gives

$$
\begin{aligned}
B^{(1)} & \leq O\left(m^{-1 / 2}\right)+\int_{\left\{t: x<F\left(t^{-}\right)<\frac{1+x}{2}\right\}} \Phi\left(\frac{\sqrt{m}\left(x-F\left(t^{-}\right)\right)}{\sigma\left(F\left(t^{-}\right)\right)}\right) d B_{r, n-r+1}(F(t)) \\
(3.1) & \leq O\left(m^{-1 / 2}\right)+\int_{\left\{t: x<F\left(t^{-}\right)<\frac{1+x}{2}\right\}} \int_{B_{t}} \chi_{B_{t}}(u) d \Phi(u) d B_{r, n-r+1}(F(t)),
\end{aligned}
$$

where $B_{t}:=\left(-\infty, \sqrt{m}\left(x-F\left(t^{-}\right)\right)\right)$, and $\chi_{B}$ is the indicator function over the event $B$. Let $A_{x}=\left\{s: x<F\left(s^{-}\right)<(1+x) / 2\right\}$. In our case, since $x \in\left(F\left(d^{-}\right), F(d)\right]$, there are two possibilities. One, $x \in\left(F\left(d^{-}\right), F(d)\right)$, or two, $x=F(d)$. In the first case $x-F\left(d^{-}\right)>0$, and Chebyshev's inequality gives the rate. Furthermore, if $x=F(d)$ and $\min _{t: F\left(t^{-}\right)>x} F\left(t^{-}\right)-x=: c_{x}>0$, then again Chebyshev's inequality will give the rate. So assume $\inf _{t: F\left(t^{-}\right)>x} F\left(t^{-}\right)=x$. Now we need to make a little more delicate argument. We use the fact that the Radon-Nikodym derivative of 
$B_{r, n-r+1} \circ F$, with respect to $F$, is bounded by a constant $K$. This follows from the fact that the density of $B_{r, n-r+1}$ is bounded. For a given value of $u<0$, let $C_{x, u}:=\left\{s: s \in A_{x}, u<\sqrt{m}\left(x-F\left(s^{-}\right)\right)\right\}$. That is, $s \in C_{x, u}$ implies that $x<F\left(s^{-}\right)<x-\frac{u}{\sqrt{m}}$. Using (3.1), we see that

$$
\begin{aligned}
& \int_{\left\{t: x<F\left(t^{-}\right)<\frac{1+x}{2}\right\}} \Phi\left(\frac{\sqrt{m}\left(x-F\left(t^{-}\right)\right)}{\sigma\left(F\left(t^{-}\right)\right)}\right) d B_{r, n-r+1}(F(t)) \\
& \quad \leq K \int_{-\infty}^{0} \int_{C_{x, u}} 1 d F(t) d \Phi(u)=K \int_{-\infty}^{0}\left(x-\frac{u}{\sqrt{m}}-x\right) d \Phi(u) \leq \frac{K E|Z|}{\sqrt{m}},
\end{aligned}
$$

after ignoring the set $D$ of Gaussian measure zero, for which $F\left(t^{-}\right)<x-u m^{-1 / 2} \leq$ $F(t)$. The other cases can be exhausted by similar arguments.

Lemma 1. For any positive integer $k$, we have the following:

$$
\begin{gathered}
\int_{(-\infty, x]}\left(F\left(u^{-}\right)\right)^{k} d F(u)=-\sum_{d \leq x} F(d)\left((F(d))^{k}-\left(F\left(d^{-}\right)\right)^{k}\right) \\
+\frac{(F(x))^{k+1}}{k+1}+\frac{k}{k+1} \sum_{d \leq x}\left((F(d))^{k+1}-\left(F\left(d^{-}\right)\right)^{k+1}\right) .
\end{gathered}
$$

This result also holds when $(-\infty, x]$ is replaced by $(-\infty, x)$ and $\left(F\left(u^{-}\right)\right)^{k}$ is replaced by $(F(u))^{k}$ on the left side and $\{d \leq x\}$ is replaced by $\{d<x\}$ and $(F(x))^{k+1}$ is replaced by $\left(F\left(x^{-}\right)\right)^{k+1}$ on the right side respectively.

Proof. We will prove the first identity, since part two follows similarly. First,

$$
\int_{(-\infty, x]}\left(F\left(u^{-}\right)\right)^{k} d F(u)+\int_{(-\infty, x]} F(u) d(F(u))^{k}=(F(x))^{k+1} .
$$

Now putting the following two expressions together gives the proof:

$$
\begin{aligned}
\int_{(-\infty, x] \cap D^{c}} F(u) d(F(u))^{k} & =\frac{k}{k+1} \int_{(-\infty, x] \cap D^{c}} 1 d(F(u))^{k+1} \\
& =\frac{k(F(x))^{k+1}}{k+1}-\frac{k}{k+1} \sum_{d \leq x}\left((F(d))^{k+1}-\left(F\left(d^{-}\right)\right)^{k+1}\right), \\
\int_{(-\infty, x] \cap D} F(u) d(F(u))^{k} & =\sum_{d \leq x} F(d)\left((F(d))^{k}-\left(F\left(d^{-}\right)\right)^{k}\right) .
\end{aligned}
$$

Proof of Theorem 2.3. By the definition of the first nontrivial record value,

$$
P\left(X_{U(2)} \leq x\right)=\sum_{k=2}^{\infty} \int_{(-\infty, x]} \int_{(-\infty, v)}(F(u))^{k-2} d F(u) d F(v) .
$$

Now we use the result of the last lemma, first on the inner integral to get

$$
\begin{gathered}
\int_{(-\infty, v)}(F(u))^{k-2} d F(u)=-\sum_{d \in D} F\left(d^{-}\right)\left((F(d))^{k-2}-\left(F\left(d^{-}\right)\right)^{k-2}\right) \chi_{(d, \infty)}(v) \\
+\frac{\left(F\left(v^{-}\right)\right)^{k-1}}{k-1}+\frac{k-2}{k-1} \sum_{d \in D}\left((F(d))^{k-1}-\left(F\left(d^{-}\right)\right)^{k-1}\right) \chi_{(d, \infty)}(v)
\end{gathered}
$$


where $\chi_{A}(v)$ is the indicator function of $A$. The last lemma gives

$$
\begin{aligned}
\int_{(-\infty, x]} & \int_{(-\infty, v)}(F(u))^{k-2} d F(u) d F(v)=\frac{1}{k-1} \int_{(-\infty, x]}\left(F\left(v^{-}\right)\right)^{k-1} d F(v) \\
& +\sum_{d \leq x}\left\{\frac{\left.(k-2)(F(d))^{k-1}-\left(F\left(d^{-}\right)\right)^{k-1}\right)}{k-1}\right\}(F(x)-F(d)) \\
& -\sum_{d \leq x} F\left(d^{-}\right)\left((F(d))^{k-2}-\left(F\left(d^{-}\right)\right)^{k-2}\right)(F(x)-F(d)),
\end{aligned}
$$

and the last integral equals

$$
\frac{(F(x))^{k}}{k(k-1)}+\sum_{d \leq x} \frac{\left((F(d))^{k}-\left(F\left(d^{-}\right)\right)^{k}\right)}{k}-\sum_{d \leq x} \frac{F(d)\left((F(d))^{k-1}-\left(F\left(d^{-}\right)\right)^{k-1}\right)}{k-1} .
$$

Now adding over $k$ gives that

$$
\begin{gathered}
P\left(X_{U(2)} \leq x\right)=H(F(x))+\sum_{d \leq x}\left\{R(F(d))-F(d)-R\left(F\left(d^{-}\right)\right)+F\left(d^{-}\right)\right\} \\
-\sum_{d \leq x} F(d)\left\{R(F(d))-R\left(F\left(d^{-}\right)\right)\right\}+\sum_{d \leq x}\left\{\frac{H(F(d))}{1-F(d)}-\frac{H\left(F\left(d^{-}\right)\right)}{1-F\left(d^{-}\right)}\right\}(F(x)-F(d)) \\
-\sum_{d \leq x} F\left(d^{-}\right)\left(\frac{1}{1-(F(d))}-\frac{1}{1-\left(F\left(d^{-}\right)\right)}\right)(F(x)-F(d)) .
\end{gathered}
$$

Combine the first terms of the first two sums, and the third and second terms of the first two sums respectively, and use $-F+(1-F) R(F)=-H(F)$, to get the final result.

The proof of the following lemma, being similar to that of Lemma 1, is omitted.

Lemma 2. When a,c are positive integers, we have the following results:

$$
\begin{array}{r}
\int_{(-\infty, x)}\left(F\left(u^{-}\right)\right)^{a}\left(1-F\left(u^{-}\right)\right)^{c} d F=\sum_{d<x}\left(F\left(d^{-}\right)\right)^{a}\left(1-F\left(d^{-}\right)\right)^{c}(\Delta F)_{d} \\
+B(a+1, c+1)\left\{B_{a+1, c+1}\left(F\left(x^{-}\right)\right)-\sum_{d<x}\left(\Delta B_{a+1, c+1}(F(.))\right)_{d}\right\} .
\end{array}
$$

The result also holds when $(-\infty, x)$ is replaced by $(-\infty, x]$ on the left side and $\{d<x\}$ is replaced by $\{d \leq x\}$ and $B_{a+1, c+1}\left(F\left(x^{-}\right)\right)$is replaced by $B_{a+1, c+1}(F(x))$ on the right side.

Proof of Theorem 2.4. It is easy to see that $R_{2}\left(x^{-}\right)$, as given in the theorem, is the Radon-Nikodym derivative of $H_{2}(x)$ with respect to $F$. Let $R_{r-1}\left(x^{-}\right)$be the derivative of $H_{r-1}$ with respect to $F$. Then by the definition of $R_{r}(x)$, we see that

$$
R_{r}(x)=\int_{(-\infty, x]} \frac{f_{r-1} \circ F\left(u^{-}\right)}{1-F(u)} d F(u)+\sum_{d<x} \sum_{k=2}^{r-1} g_{k}(d) h_{r-k+2}(x, d) .
$$


Since $h_{r-k+2}(x, x)=0$, we may take the outer sum as $\sum_{d \leq x}$. The integral term can be evaluated as follows.

$$
\begin{aligned}
\int_{(-\infty, x] \cap D^{c}} \frac{f_{r-1} \circ F\left(u^{-}\right)}{1-F(u)} d F(u) & =\int_{(-\infty, x]} 1 d f_{r} \circ F(u)-\int_{(-\infty, x] \cap D} 1 d f_{r} \circ F(u) \\
& =f_{r} \circ F(x)-\sum_{d \leq x}\left(\Delta f_{r} \circ F\right)_{d} .
\end{aligned}
$$

Putting the terms together, and using induction, we get that $R_{r}\left(x^{-}\right)$is the RadonNikodym derivative of $H_{r}(x)$. To get $H_{r}$, note that

$$
H_{r}(x)=\int_{(-\infty, x]} f_{r} \circ F\left(u^{-}\right) d F(u)+\sum_{d \leq x} \sum_{k=2}^{r} g_{k}(d) \int_{(d, x]} h_{r-k+2}\left(u^{-}, d\right) d F(u) .
$$

Finally, $H_{r}$ is obtained since

$$
\begin{aligned}
& \int_{(-\infty, x]} f_{r} \circ F\left(u^{-}\right) d F(u) \\
& \quad=\int_{(-\infty, x]} 1 d \phi_{r} \circ F(u)-\int_{(-\infty, x] \cap D} 1 d \phi_{r} \circ F(u)+\sum_{d \leq x} f_{r} \circ F\left(d^{-}\right)(\Delta F)_{d} \\
& \quad=\phi_{r} \circ F(x)+\sum_{d \leq x}\left\{f_{r} \circ F\left(d^{-}\right)(\Delta F)_{d}-\left(\Delta \phi_{r} \circ F\right)_{d}\right\} \cdot \square
\end{aligned}
$$

Proof of Theorem 2.5. With one exception, the proof of this theorem remains identical to that of Theorem 2.2, the exception being that the derivative of $B_{r, n-r+1} \circ F$ remained bounded, while the derivative of $H_{r}$, namely $R_{r}\left(x^{-}\right)$, need not be bounded over $\Re$. The patch up requires noting that the proof of Theorem 2.2 needs boundedness over regions such as $(-\infty, t]$ where $F(t)<1$, and $R_{r}(t)$ is nonnegative, nondecreasing, and hence it also obeys this condition.

Proof of Theorem 2.6. Let $\left(\Delta F^{k}\right)_{d}=F(d)^{k}-F\left(d^{-}\right)^{k}$. By the definition,

$$
\begin{aligned}
& \frac{P\left(S_{m}^{*}=\ell\right)}{\left(\begin{array}{c}
m \\
\ell
\end{array}\right)}=\sum_{k=2}^{\infty} \int_{\Re}\left(F\left(v^{-}\right)\right)^{\ell}\left(1-F\left(v^{-}\right)\right)^{m-\ell} d F(v) \int_{(-\infty, v)}(F(u))^{k-2} d F(u) \\
& =\sum_{k=2}^{\infty} \int_{\Re}\left(F\left(v^{-}\right)\right)^{\ell}\left(1-F\left(v^{-}\right)\right)^{m-\ell} \frac{\left(F\left(v^{-}\right)\right)^{k-1}}{k-1} d F(v) \\
& \quad+\sum_{k=2}^{\infty} \int_{\Re}\left(F\left(v^{-}\right)\right)^{\ell}\left(1-F\left(v^{-}\right)\right)^{m-\ell} \frac{k-2}{k-1} \sum_{d \in D}\left(\Delta F^{k-1}\right)_{d} \chi_{(d, \infty)}(v) d F(v) \\
& \quad-\sum_{k=2}^{\infty} \int_{\Re}\left(F\left(v^{-}\right)\right)^{\ell}\left(1-F\left(v^{-}\right)\right)^{m-\ell} \sum_{d \in D} F\left(d^{-}\right)\left(\Delta F^{k-2}\right)_{d} \chi_{(d, \infty)}(v) d F(v) .
\end{aligned}
$$

Denote the above three terms as $A, B, C$. For $B, C$, we interchange the two summation signs and use Lemma 2 and then rewrite $B-C$ as

$$
\begin{gathered}
\sum_{d \in D}\left(\frac{H(F(d))-F\left(d^{-}\right)}{1-F(d)}-\frac{H\left(F\left(d^{-}\right)\right)-F\left(d^{-}\right)}{1-F\left(d^{-}\right)}\right)[B(\ell+1, m-\ell+1)\{1- \\
\left.\left.\beta_{\ell}(F(d))-\sum_{\delta>d}\left(\Delta \beta_{\ell}(F(.))\right)_{\delta}\right\}+\sum_{\delta>d}\left(F\left(\delta^{-}\right)\right)^{\ell}\left(1-F\left(\delta^{-}\right)\right)^{m-\ell}(\Delta F)_{\delta}\right],
\end{gathered}
$$


where $\beta_{\ell}(x)=B_{\ell+1, m-\ell+1}(x)$. A similar argument gives that

$$
\begin{aligned}
A= & \sum_{k=2}^{\infty} \frac{1}{k-1}\left[B(k+\ell, m-\ell+1)\left\{1-\sum_{d \in D}\left(\Delta B_{k+\ell, m-\ell+1}(F(.))\right)_{d}\right\}\right. \\
& \left.+\sum_{d \in D}\left(F\left(d^{-}\right)\right)^{k-1}\left(F\left(d^{-}\right)\right)^{\ell}\left(1-F\left(d^{-}\right)\right)^{m-\ell}(\Delta F)_{d}\right] \\
= & \int_{0}^{1} u^{\ell}(1-u)^{m-\ell} d \phi_{2}(u)-\sum_{d \in D} \int_{F\left(d^{-}\right)}^{F(d)} u^{\ell}(1-u)^{m-\ell} d \phi_{2}(u) \\
& +\sum_{d \in D}\left(F\left(d^{-}\right)\right)^{\ell}\left(1-F\left(d^{-}\right)\right)^{m-\ell}(\Delta F)_{d} R\left(F\left(d^{-}\right)\right) .
\end{aligned}
$$

Using the fact that $H(w)-w=-R(w)(1-w)$, and then putting $A, B-C$ together finishes the proof.

\section{ACKNOWLEDGMENTS}

We would like to thank Professor Cihan Orhan for his support. The second author was supported by TUBITAK while visiting Ankara University, Turkey.

\section{REFERENCES}

1. M. Ahsanullah, Record Statistics, Nova Science Publishers, Inc., Cormack, New York, 1995. MR.1443904 (98d:62088)

2. B. Arnold, N. Balakrishnan, and H. N. Nagaraja, Records, John Wiley \& Sons, New York, 1998. MR 1628157 (2000b:60127)

3. I. G. Bairamov, Some distribution free properties of statistics based on record values and characterizations of the distributions through a record, J. Appl. Statist. Sci. 5 (1997), no. 1, 17-25. MR 1439625 (98d:62016)

4. I. G. Bairamov and S. N. Eryilmaz, Distributional properties of statistics based on minimal spacing and record exceedance statistics, J. Statist. Plann. Infer. 90 (2000), 21-23. MR.1791579 (2002f:62052)

5. I. G. Bairamov, O. L. Gebizlioglu and M. F. Kaya, Asymptotic Distributions of Statistics Based on Order Statistics and Record Values and Invariant Confidence Intervals, In "Asymptotic Methods in Probability and Statistics with Applications" (Eds. N. Balakrishnan; I. A. Ibragimov, and V. B. Nevzorov), 309-320, Birkhäuser, Boston 2001. MR.1890335

6. I. G. Bairamov and S. Kotz, On distributions of exceedances associated with order statistics and record values for arbitrary distributions, Statistical Papers, 42 (2001), 171-185. MR 1836847 (2002c:62086)

7. I. G. Bairamov and Yu. I. Petunin, Structure of invariant confidence intervals containing the main distributed mass, Theorya Veroyatnostey i ee Primeneniya, 35 (1990) no. 1, 15-26 (English translation in Theor. Probab. Appl., 35 (1991), no. 1, 15-26). MR1050050 (91m:62048)

8. H. A. David, Order Statistics. Second edition, John Wiley and Sons, New York, 1981. MR 0597893 (82i:62073)

9. B. Epstein, Tables for the distribution of the number of exceedances, Ann. Math. Stat., 25 (1954), 762-768. MR0065074 (16:380f)

10. E. J. Gumbel, (1954a). Statistical theory of extreme values and some practical applications, National Bureau of Standards, Washington (1954a). MR0061342 (15:811b)

11. E. J. Gumbel, Elementare Ableitung der Momente für die Zahl der Überschreitungen, Mitteilungsblatt für Math. Stat, 6 (1954b), 164-169. MR0064357 (16:270e)

12. E. Gumbel, and H. von Schelling, The distribution of the number of exceedances, Ann. Math. Stat., 21 (1950), 247-262. MR0035415 (11:732d)

13. N. Johnson, and S. Kotz, Some generalizations of Bernoulli and Polya-Eggenberger contagion models, Statist. Papers., 32 (1991) 1-17. MR1123680 (92g:62020) 
14. N. Johnson, and S. Kotz, Further comments on Matveychuk and Petunin's generalized Bernoulli model, and nonparametric tests of homogeneity, J. Statist. Plann. Infer., 41 (1994), 61-72. MR 1292147 (95g:62105)

15. W. Katzenbeisser, The distribution of two-sample location exceedance test statistics under Lehmann alternatives, Statist. Hefte, 26 (1985), 131-138. MR0861796

16. W. Katzenbeisser, The exact power of two-sample location tests based on exceedances against shift alternatives, Math. Op. Forsch. (Statistics)., 20 (1986), 47-54. MR0995233 (90i:62030)

17. S. A. Matveřchuk, and Yu. I. Petunin, A generalization of the Bernoulli model arising in order statistics, I, Ukrain. Math. J., 42 (1990), 518-528. MR1051875 (91j:62056)

18. S. A. Matverchuk, and Yu. I. Petunin, A generalization of the Bernoulli model arising in order statistics, II, Ukrain. Math. J., 43 (1991), 779-785. MR.1134403 (92m:62055)

19. K. Sarkadi, On the distribution of the number of exceedances, Ann. Math. Stat., 28 (1957), 1021-1023.

20. J. Wesolowski, and M. Ahsanullah, (1998). Distributional properties of exceedance statistics, Ann. Inst. Statist. Math., 50 (1998), no. 3, 543-565. MR1664524 (2000d:62020)

Department of Mathematics, Izmir University of Economics, Izmir, Turkey

Department of Mathematical Sciences, Kent State University, Kent, Ohio, 44242

E-mail address: kazim@math.kent.edu 